\title{
A SUFFICIENT CONDITION FOR THE EXISTENCE OF AN INVARIANT PROBABILITY MEASURE FOR MARKOV PROCESSES
}

\author{
O. L. V. COSTA, ${ }^{*}$ Universidade de São Paulo \\ F. DUFOUR, ${ }^{* *}$ Université Bordeaux I and Université Bordeaux IV
}

\begin{abstract}
In this paper, it is shown that the Foster-Lyapunov criterion is sufficient to ensure the existence of an invariant probability measure for both discrete- and continuous-time Markov processes without any additional hypotheses (such as irreducibility).
\end{abstract}

Keywords: Markov process; invariant probability measure; Foster-Lyapunov criterion; petite set; discrete time; continuous time

2000 Mathematics Subject Classification: Primary 60J10

Secondary 93E15

\section{Introduction}

In the last 30 years, a great deal of attention has been paid to the existence of invariant probability measures for Markov chains and for continuous-time Markov processes. In general, it is not a simple matter to determine whether a given Markov process on a general state space has an invariant probability measure. For discrete-time Markov chains, a rather complete discussion on this subject is available in [8].

Sufficient conditions for the existence of an invariant probability measure based on a FosterLyapunov-type criterion (see (2.2) and (2.3), below) have been presented in the literature for both the discrete- and continuous-time cases. This Foster-Lyapunov criterion, also known as the drift condition, is written in terms of a set $C$, called the 'test' set. In addition to satisfying the Foster-Lyapunov criterion, these sufficient conditions require an assumption on the test set $C$ and other additional hypotheses. For example, some of the hypotheses that have been considered for the existence of an invariant probability measure for the discrete-time case are as follows.

- The set $C$ is compact. In this case, Tweedie proved the result in [10], with the additional condition that the Markov chain is a $T$-chain. The result also holds if, instead of being a $T$-chain, the chain is weak Feller (see [10] or [8, p. 296]). For weak Feller chains it is worth mentioning that there are some necessary and sufficient conditions for the existence of an invariant probability measure not requiring the Foster-Lyapunov criterion (see, for example, [4] and the references therein).

Received 20 December 2004; revision received 11 April 2005.

* Postal address: Departamento de Engenharia de Telecomunicações e Controle, Escola Politécnica da Universidade de São Paulo, São Paulo, 05508 900, Brazil. Email address: oswaldo@lac.usp.br

** Postal address: Mathématiques Appliqées de Bordeaux, Universite Bordeaux I, 351 cours de la Liberation, 33405

Talence Cedex, France. Email address: dufour@math.u-bordeaux1.fr 
- The set $C$ satisfies a uniform countable-additivity condition (see [11, Equation (4)]). In this case, Tweedie proved the existence of an invariant probability measure in [11].

- The set $C$ is 'petite' (see Definition 2.5, below) and the chain is irreducible. As discussed by Tweedie [11, p. 347], with the set $C$ petite, the Foster-Lyapunov criterion is known to ensure the existence of an invariant probability measure when the chain is irreducible. This was shown in [9] for a small set $C$, and was generalized to a petite set in [8].

To the best of the authors' knowledge, the most general sufficient condition for the existence of an invariant probability measure, written in terms of the Foster-Lyapunov criterion and a petite test set $C$, requires the additional hypothesis that the chain is irreducible. The aim of this paper is to remove this additional hypothesis. We extend the existing results in the literature by showing that if the drift condition is satisfied for a petite test set $C$, then there exists an invariant probability measure (without any additional hypotheses such as irreducibility). It must be pointed out that, in the discrete-time case, the authors proved that this drift condition also becomes necessary if the Markov chain is assumed to be a $T^{\prime}$-chain [2]. Here, we obtain results for a discrete-time Markov chain evolving on an arbitrary space with countably generated $\sigma$-field and for a continuous-time (Borel right, nonexplosive) Markov process evolving on a locally compact, separable metric space equipped with its Borel $\sigma$-field.

Finally, we note that, by using [11, Lemma 5], it is easy to show that if the FosterLyapunov criterion is satisfied for a set satisfying the uniform countable-additivity condition (see [11, Equation (4)]), then it is also necessarily satisfied for a petite test set. Therefore, apparently, for the purposes of ensuring the existence of an invariant probability measure, the drift condition (2.2), below, for a petite test set $C$ is more general than the uniform countable-additivity condition.

The paper is organized in the following way. In Section 2, we present the main definitions that will be required throughout the paper. In Section 3, we present the main results for the discrete-time case. The continuous-time case is considered in Section 4.

\section{Definitions}

Denote the set of positive real numbers by $\mathbb{R}_{+}$, the set of positive integers by $\mathbb{N}$, and let $\mathbb{R}_{+}^{*}:=\mathbb{R}_{+} \backslash\{0\}$ and $\mathbb{N}^{*}:=\mathbb{N} \backslash\{0\}$. Let $(\mathbb{X}, \mathscr{B})$ be a measurable space. For any $A \in \mathscr{B}$, $\mathbf{1}_{A}(x)$ denotes the indicator function associated with $A$. In this paper, we consider two types of time-homogeneous Markov process: a discrete-time Markov chain $\left\{\Psi_{n}\right\}_{n \in \mathbb{N}}$ with associated transition probability function denoted by $G$, and a continuous-time Markov process $\left\{X_{t}\right\}_{t \in \mathbb{R}_{+}}$ with associated transition semigroup denoted by $\left\{P^{t}\right\}_{t \in \mathbb{R}_{+}}$. For the latter, for every $t \in \mathbb{R}_{+}$we shall write $P^{t}(x, A)=\mathrm{P}_{x}\left(X_{t} \in A\right)$, where $x \in \mathbb{X}$ is the initial condition (i.e. $X_{0}=x$ ) and $A \in \mathcal{B}$.

We now recall some classical definitions related to Markov processes. For a complete exposition on the subject, the reader is referred to the book of Meyn and Tweedie [8], or the papers [5], [6], and [7].

Definition 2.1. A set $E \in \mathscr{B}$ is called absorbing for $\left\{X_{t}\right\}_{t \in \mathbb{R}_{+}}$or for $\left\{\Psi_{n}\right\}_{n \in \mathbb{N}}$ if $E \neq \varnothing$ and if $P^{t}(x, E)=1$ for all $x \in E$ and $t \in \mathbb{R}_{+}$, or, respectively, $G(x, E)=1$ for all $x \in E$.

Definition 2.2. A set $E \in \mathscr{B}$ is called closed for $\left\{X_{t}\right\}_{t \in \mathbb{R}_{+}}$or for $\left\{\Psi_{n}\right\}_{n \in \mathbb{N}}$ if $E \neq \varnothing$ and if $\mathrm{P}_{x}\left(X_{t} \in E\right.$ for all $\left.t \in \mathbb{R}_{+}\right)=1$, or, respectively, $\mathrm{P}_{x}\left(\Psi_{n} \in E\right.$ for all $\left.n \in \mathbb{N}\right)=1$. 
Definition 2.3. If $b=\left\{b_{k}\right\}_{k=0}^{\infty}$ is a sequence of real numbers defining a probability on $\mathbb{N}$, then the stochastic kernel $K_{b}^{\Psi}$ associated with the discrete-time Markov chain $\left\{\Psi_{k}\right\}_{k \in \mathbb{N}}$ is defined on $\mathbb{X} \times \mathscr{B}$ by

$$
K_{b}^{\Psi}(x, A):=\sum_{k=0}^{\infty} b_{k} G^{k}(x, A) \quad \text { for all } x \in \mathbb{X} \text { and all } A \in \mathscr{B}
$$

Definition 2.4. If $F$ is a probability distribution on $\mathbb{R}_{+}$, then the stochastic kernel $K_{F}^{X}$ associated with the continuous-time Markov process $\left\{X_{t}\right\}_{t \in \mathbb{R}_{+}}$is defined on $\mathbb{X} \times \mathscr{B}$ by

$$
K_{F}^{X}(x, A):=\int_{0}^{\infty} P^{t}(x, A) F(\mathrm{~d} t) \quad \text { for all } x \in \mathbb{X} \text { and all } A \in \mathscr{B} .
$$

The resolvent associated with the transition semigroup $\left\{P^{t}\right\}_{t \in \mathbb{R}_{+}}$is defined by

$$
R(x, A):=\int_{0}^{\infty} P^{t}(x, A) \mathrm{e}^{-t} \mathrm{~d} t .
$$

Definition 2.5. A set $C \in \mathscr{B}$ is called a petite set for $\left\{X_{t}\right\}_{t \in \mathbb{R}_{+}}$or for $\left\{\Psi_{n}\right\}_{n \in \mathbb{N}}$ if there exist a probability distribution $F$ on $\mathbb{R}_{+}^{*}$ and a nontrivial measure $v$ on $(\mathbb{X}, \mathscr{B})$ such that $K_{F}^{X}(x, A) \geq v(A)$ for all $A \in \mathscr{B}$ and all $x \in C$, or, respectively, there exist a probability $b$ on $\mathbb{N}^{*}$ and a nontrivial measure $v$ on $(\mathbb{X}, \mathscr{B})$ such that $K_{b}^{\Psi}(x, A) \geq v(A)$ for all $A \in \mathscr{B}$ and all $x \in C$.

Definition 2.6. For a discrete-time Markov chain $\left\{\Psi_{n}\right\}_{n \in \mathbb{N}}$, the first hitting time $\tau_{A}^{\Psi}$ of the set $A \in \mathscr{B}$ is defined as

$$
\tau_{A}^{\Psi}:=\inf \left\{n \geq 1: \Psi_{n} \in A\right\} .
$$

We also define $L^{\Psi}(x, A):=\mathrm{P}_{x}\left(\tau_{A}^{\Psi}<\infty\right)$ for all $(x, A) \in \mathbb{X} \times \mathscr{B}$.

We next define the extended generator $\mathcal{A}$ for $\left\{X_{t}\right\}_{t \in \mathbb{R}_{+}}$, and its domain $D(\mathcal{A})$.

Definition 2.7. Define $D(\mathcal{A})$ to be the set of all measurable functions $V: \mathbb{X} \times \mathbb{R}_{+} \rightarrow \mathbb{R}$ for which there exists a measurable function $U: \mathbb{X} \times \mathbb{R}_{+} \rightarrow \mathbb{R}$ such that, for each $x \in \mathbb{X}$ and $t>0$,

$$
\begin{aligned}
\mathrm{E}_{x}\left[\int_{0}^{t} U\left(X_{s}, s\right) \mathrm{d} s\right] & <\infty, \\
\mathrm{E}_{x}\left[V\left(X_{t}, t\right)\right] & =V(x, 0)+\mathrm{E}_{x}\left[\int_{0}^{t} U\left(X_{s}, s\right) \mathrm{d} s\right] .
\end{aligned}
$$

The set $D(\mathcal{A})$ is referred to as the domain of the extended generator $\mathcal{A}$ for $\left\{X_{t}\right\}_{t \in \mathbb{R}_{+}}$. This generator is defined over $D(\mathcal{A})$ as follows: $\mathcal{A} V:=U$ for $V \in D(\mathcal{A})$.

We conclude this section by presenting the Foster-Lyapunov criterion. For a discrete-time Markov chain with transition probability function $G$, this criterion can be stated as follows.

Condition 2.1. (Foster-Lyapunov criterion: discrete-time case.) There exist an extendedvalued nonnegative measurable function $V$ defined on $\mathbb{X}$, with $V\left(x_{0}\right)<\infty$ for at least one $x_{0} \in \mathbb{X} ;$ a set $C$; and a constant $d \in \mathbb{R}_{+}$such that

$$
G V(x) \leq V(x)-1+d \mathbf{1}_{C}(x)
$$


For a continuous-time Markov process with extended generator $\mathcal{A}$, the Foster-Lyapunov criterion instead has the following statement.

Condition 2.2. (Foster-Lyapunov criterion: continuous-time case.) There exist a nonnegative measurable function $W \in D(\mathcal{A})$, a set $C$, and a constant $d \in \mathbb{R}_{+}$such that

$$
\mathcal{A} W(x) \leq-1+d \mathbf{1}_{C}(x)
$$

\section{The discrete-time case}

In this section, it is assumed that the $\sigma$-field $\mathcal{B}$ associated with the state space $\mathbb{X}$ is countably generated. We show that if the Foster-Lyapunov criterion is satisfied for a petite test set $C$, then an invariant probability measure exists.

Theorem 3.1. Suppose that the Markov chain $\left\{\Psi_{n}\right\}_{n \in \mathbb{N}}$ satisfies the Foster-Lyapunov criterion (2.2) for a petite set $C$ and for every $x$ in $\mathbb{X}$. Then there exists an invariant probability measure for $\left\{\Psi_{n}\right\}_{n \in \mathbb{N}}$.

Proof. By hypothesis, the set $F \in \mathscr{B}$ defined by $F:=\{x \in \mathbb{X}: V(x)<\infty\}$ is nonempty and, from [8, Lemma 11.3.6], is an absorbing set for the Markov chain $\left\{\Psi_{n}\right\}_{n \in \mathbb{N}}$. From [8, Theorem 11.3.4],

$$
\mathrm{E}_{x}\left[\tau_{C}^{\Psi}\right] \leq V(x)+b \mathbf{1}_{C}(x)
$$

and, thus, for all $x \in F$, we have $\mathrm{E}_{x}\left[\tau_{C}^{\Psi}\right]<\infty$, meaning that $L^{\Psi}(x, C)=1$ for all $x \in F$. Define $D:=F \cap C$. From the fact that $F$ is absorbing, it follows that $L^{\Psi}(x, D)=L^{\Psi}(x, C)=1$ for all $x \in F$, showing that $D \neq \varnothing$. Since $C$ is a petite set for the Markov chain $\left\{\Psi_{n}\right\}_{n \in \mathbb{N}}$, there exist a probability $b$ on $\mathbb{N}^{*}$ and a nontrivial measure $v$ on $\mathscr{B}$ such that $K_{b}^{\Psi}(x, A) \geq v(A)$ for all $x \in C$ and all $A \in \mathscr{B}$. In particular, for $x \in D$, we have $K_{b}^{\Psi}(x, \mathbb{X} \backslash F)=0$, meaning that $v(\mathbb{X} \backslash F)=0$ and $v(F)=v(\mathbb{X})>0$. Let us denote by $\bar{G}$ the Markov kernel corresponding to $G$ restricted to $F$, and by $\left\{\bar{\Psi}_{n}\right\}_{n \in \mathbb{N}}$ the Markov chain associated with $\bar{G}$. Consequently, it follows from the previous discussion that $D$ is a petite set for $\left\{\bar{\Psi}_{n}\right\}_{n \in \mathbb{N}}$. Now, by combining the fact that $L^{\bar{\Psi}}(x, D)=1$ for all $x \in F$ with [5, Theorem 4.1(i)], we find that the Markov chain $\left\{\bar{\Psi}_{n}\right\}_{n \in \mathbb{N}}$ is Harris recurrent and satisfies

$$
\bar{G} V(x) \leq V(x)-1+d \mathbf{1}_{D}(x) \text { for all } x \in H .
$$

By applying [8, Theorem 11.0.1], from this we find that there exists an invariant probability measure $\pi$ for the Markov chain $\left\{\bar{\Psi}_{n}\right\}_{n \in \mathbb{N}}$ and, consequently, for the Markov chain $\left\{\Psi_{n}\right\}_{n \in \mathbb{N}}$. This completes the proof.

\section{The continuous-time case}

In this section, it is assumed that the state space $\mathbb{X}$ is a locally compact and separable metric space equipped with its Borel field $\mathscr{B}(\mathbb{X})$, and that $\left\{X_{t}\right\}_{t \in \mathbb{R}_{+}}$is a nonexplosive, Borel right process. Following the framework of [3], [6], and [7], and as defined in Section 2, $\left\{X_{t}\right\}_{t \in \mathbb{R}_{+}}$ is a time-homogeneous Markov process with state space $(\mathbb{X}, \mathscr{B}(\mathbb{X}))$ and associated transition semigroup $\left\{P^{t}\right\}_{t \in \mathbb{R}_{+}}$. Recall that, for every $t \in \mathbb{R}_{+}$, we have $P^{t}(x, A)=\mathrm{P}_{x}\left(X_{t} \in A\right)$, where $x \in \mathbb{X}$ is the initial condition, $\mathcal{A}$ represents the extended generator of $\left\{X_{t}\right\}_{t \in \mathbb{R}_{+}}, D(\mathcal{A})$ represents the domain of the extended generator (see Definition 2.7), and $R$ represents the resolvent associated with the transition semigroup $\left\{P^{t}\right\}_{t \in \mathbb{R}_{+}}$(see (2.1) and Definition 2.4).

Since $R$ is a transition probability function, we can define $\left\{\Psi_{n}^{R}\right\}_{n \in \mathbb{N}}$ to be the Markov chain associated with $R$. 
Theorem 4.1. Suppose that $\left\{X_{t}\right\}_{t \in \mathbb{R}_{+}}$is a nonexplosive, Borel right process satisfying the Foster-Lyapunov criterion (2.3) for a petite set $C$ and for every $x$ belonging to a closed set $H$. Then there exists an invariant probability measure for $\left\{X_{t}\right\}_{t \in \mathbb{R}_{+}}$.

Proof. Let $C$ be a petite set, let $H$ be a closed set for $\left\{X_{t}\right\}_{t \in \mathbb{R}_{+}}$, let $W: \mathbb{X} \rightarrow \mathbb{R}_{+}$be a measurable function in $D(\mathcal{A})$, and let $d \in \mathbb{R}_{+}$be a constant such that (2.3) is satisfied for every $x \in H$. By using the comparison theorem [7, Theorem 1.1] and the fact that $H$ is closed, we find that

$$
\mathrm{e}^{-t} P^{t} W(x) \leq-t \mathrm{e}^{-t}+\mathrm{e}^{-t} W(x)+d \mathrm{e}^{-t} \int_{0}^{t} P^{s}(x, C) \mathrm{d} s \quad \text { for all } x \in H .
$$

By integrating (4.1) and using the fact that $H$ is absorbing for the Markov chain $\left\{\Psi_{n}^{R}\right\}_{n \in \mathbb{N}}$, from this we find that

$$
R W(x) \leq-1+W(x)+d R(x, C \cap H) \text { for all } x \in H .
$$

There exist a constant $\alpha>0$ and a petite set $D$ for the Markov chain $\left\{\Psi_{n}^{R}\right\}_{n \in \mathbb{N}}$ such that

$$
R W(x) \leq-\alpha+W(x)+d \mathbf{1}_{D}(x) \text { for all } x \in H .
$$

If $C \cap H=\varnothing$ then this inequality is trivially satisfied. If $C \cap H \neq \varnothing$ then, by following the same arguments as in the proof of [3, Theorem 5.1], we easily find from (4.2) that (4.3) is satisfied.

Define the extended-valued nonnegative function $V$ on $\mathbb{X}$ as

$$
V(x):= \begin{cases}\frac{1}{\alpha} W(x) & \text { for } x \in H, \\ \infty & \text { for } x \in H^{\mathrm{c}} .\end{cases}
$$

Set $\bar{d}=d / \alpha$. Since $H$ is absorbing for the Markov chain $\left\{\Psi_{n}^{R}\right\}_{n \in \mathbb{N}}$, we find from (4.3) that

$$
R V(x) \leq-1+V(x)+\bar{d} \mathbf{1}_{D}(x) \text { for all } x \in \mathbb{X} .
$$

From Theorem 3.1, it follows that there exists an invariant probability measure for $\left\{\Psi_{n}^{R}\right\}_{n \in \mathbb{N}}$ and, consequently, for $\left\{X_{t}\right\}_{t \in \mathbb{R}_{+}}$, by using [1, Lemma 1].

\section{Acknowledgements}

The authors would like to thank the referee for several very helpful comments and suggestions. This work was supported by CAPES/COFECUB grant 425/03. The first author received financial support from CNPq (the Brazilian National Research Council), grants 472920/03-0 and 304866/03-2; FAPESP (the Research Council of the State of São Paulo), grant 03/06736-7; PRONEX, grant 015/98; and IM-AGIMB.

\section{References}

[1] Azéma, J., Kaplan-Duflo, M. and Revuz, D. (1967). Mesure invariante sur les classes récurrentes des processus de Markov. Z. Wahrscheinlichkeitsth. 8, 157-181.

[2] Costa, O. L. V. And Dufour, F. (2005). On the ergodic decomposition for a class of Markov chains. Stoch. Process. Appl. 115, 401-415.

[3] Down, D., Meyn, S. P. And Tweedie, R. L. (1995). Exponential and uniform ergodicity of Markov processes. Ann. Prob. 23, 1671-1691. 
[4] Lasserre, J. B. (1997). Invariant probabilities for Markov chains on a metric space. Statist. Prob. Lett. 34, 259-266.

[5] Meyn, S. P. and Tweedie, R. L. (1992). Stability of Markovian processes. I. Criteria for discrete-time chains. Adv. Appl. Prob. 24, 542-574.

[6] Meyn, S. P. and Tweedie, R. L. (1993). Stability of Markovian processes. II. Continuous-time processes and sampled chains. Adv. Appl. Prob. 25, 487-517.

[7] Meyn, S. P. And Tweedie, R. L. (1993). Stability of Markovian processes. III. Foster-Lyapunov criteria for continuous-time processes. Adv. Appl. Prob. 25, 518-548.

[8] Meyn, S. P. And Tweedie, R. L. (1993). Markov Chains and Stochastic Stability. Springer, Berlin.

[9] Tweedie, R. L. (1976). Criteria for classifying general Markov chains. Adv. Appl. Prob. 8, 737-771.

[10] TweEdie, R. L. (1988). Invariant measures for Markov chains with no irreducibility assumptions. In $A$ Celebration of Applied Probability (J. Appl. Prob. Spec. Vol. 25A), Applied Probability Trust, Sheffield, pp. 275285.

[11] Tweedie, R. L. (2001). Drift conditions and invariant measures for Markov chains. Stoch. Process. Appl. 92, 345-354. 